\title{
Interactive comment on "Spatial and temporal variations in plant Water Use Efficiency inferred from tree-ring, eddy covariance and atmospheric observations" by Margriet Groenendijk et al.
}

Margriet Groenendijk et al.

s.c.dekker@uu.nl

Received and published: 7 May 2016

See the attached pdf for our detailed respons

Please also note the supplement to this comment:

http://www.earth-syst-dynam-discuss.net/esd-2016-4/esd-2016-4-AC1-

supplement.pdf

Interactive comment on Earth Syst. Dynam. Discuss., doi:10.5194/esd-2016-4, 2016. 